\title{
Working memory deficits after resection of the dorsolateral prefrontal cortex predicted by functional magnetic resonance imaging and electrocortical stimulation mapping
}

\author{
Case report
}

\author{
Kuan H. Kho, M.D., ${ }^{1-3}$ Geert-Jan M. RutTen, M.D., Ph.D., ${ }^{1,4}$ \\ Frans S. S. LeiJTen, M.D., Ph.D., ${ }^{2}$ ArJen van der SchaAf, Ph.D., ${ }^{3,5}$ \\ Peter C. van Rijen, M.D., Ph.D., ${ }^{1}$ ANd Nick F. Ramsey, Ph.D. ${ }^{3}$ \\ Departments of ${ }^{1}$ Neurosurgery, ${ }^{2}$ Clinical Neurophysiology, and ${ }^{3}$ Psychiatry, Rudolf Magnus Institute \\ of Neuroscience, University Medical Center Utrecht; ${ }^{4}$ Department of Neurosurgery, St. Elisabeth \\ Hospital, Tilburg; and ${ }^{5}$ Department of Radiation Oncology, University Medical Center Groningen, \\ The Netherlands
}

\begin{abstract}
$\checkmark$ Electrocortical stimulation mapping (ESM) is the clinical standard for localizing critical sensorimotor and language functions, but other functions can be assessed with this technique as well. The authors describe an 8-year-old girl with a left frontal desmoplastic gangliocytoma and medically intractable epilepsy who underwent a chronic invasive recording using electrode grids. Prior to electrode implantation, functional magnetic resonance (fMR) imaging was performed using a research protocol that included a working memory task. The ESM procedure interfered with working memory at a dorsolateral prefrontal site as predicted by fMR imaging, but because this site was part of the epileptogenic region, it was included in the resection. Since the operation the patient has been seizure free and her overall cognitive performance has improved. Yet she shows a selective impairment in working memory tasks that has persisted for over two years, indicating that the area identified using fMR imaging and ESM was critically involved in working memory. Her performance did improve, however, suggesting that compensatory mechanisms took place. This case reveals an important and perhaps critical function of the dorsolateral prefrontal cortex. Work continues to assess the specific cognitive functions subserved by the region identified with fMR imaging and ESM.
\end{abstract}

\section{KEY Words - epilepsy - dorsolateral prefrontal cortex • working memory • electrocortical stimulation mapping • functional magnetic resonance imaging • pediatric neurosurgery}

$\mathrm{I}$ $\mathrm{N}$ neurosurgery, ESM is considered the gold standard for localizing sensorimotor and language functions. ${ }^{13} \mathrm{Al}-$ though preservation of ESM-detected motor and language areas correlates with good postoperative functional outcome, it is unknown whether these areas are truly essential for the function that is being tested because ESM-detected areas are usually not resected. Other cortical functions that have been studied infrequently with ESM include vision, verbal short-term memory, ${ }^{14}$ calculation, and finger recognition. ${ }^{19}$ Electrocortical stimulation mapping has incidentally resulted in body schema disturbances and even "psychical" or experiential phenomena., ${ }^{2,15}$

We report on an epilepsy patient in whom activation of the dorsolateral prefrontal cortex was seen on fMR imaging

Abbreviations used in this paper: $\mathrm{ESM}=$ electrocortical stimulation mapping; $\mathrm{fMR}=$ functional magnetic resonance. during a working memory task. Electrocortical stimulation of the same cortical area during intracranial electroencephalography recording induced working memory deficits. We have shown previously that ESM of cortical areas identified with fMR imaging can selectively disrupt working memory. ${ }^{17}$ In this patient, however, this area was also found to be part of the epileptogenic region and was included in the resection, resulting in a postoperative deficit in specific working memory tasks.

Working memory holds a key position in models of cognitive information processing, as "a system for the temporary holding and manipulation of information during the performance of a range of cognitive tasks such as comprehension, learning and reasoning." A distributed network of frontal and parietal areas is implied, in particular the dorsolateral prefrontal cortex. ${ }^{7,8,16}$ Working memory areas are not considered part of the "eloquent" cortex in neurosurgical practice and are thus not localized by ESM. 

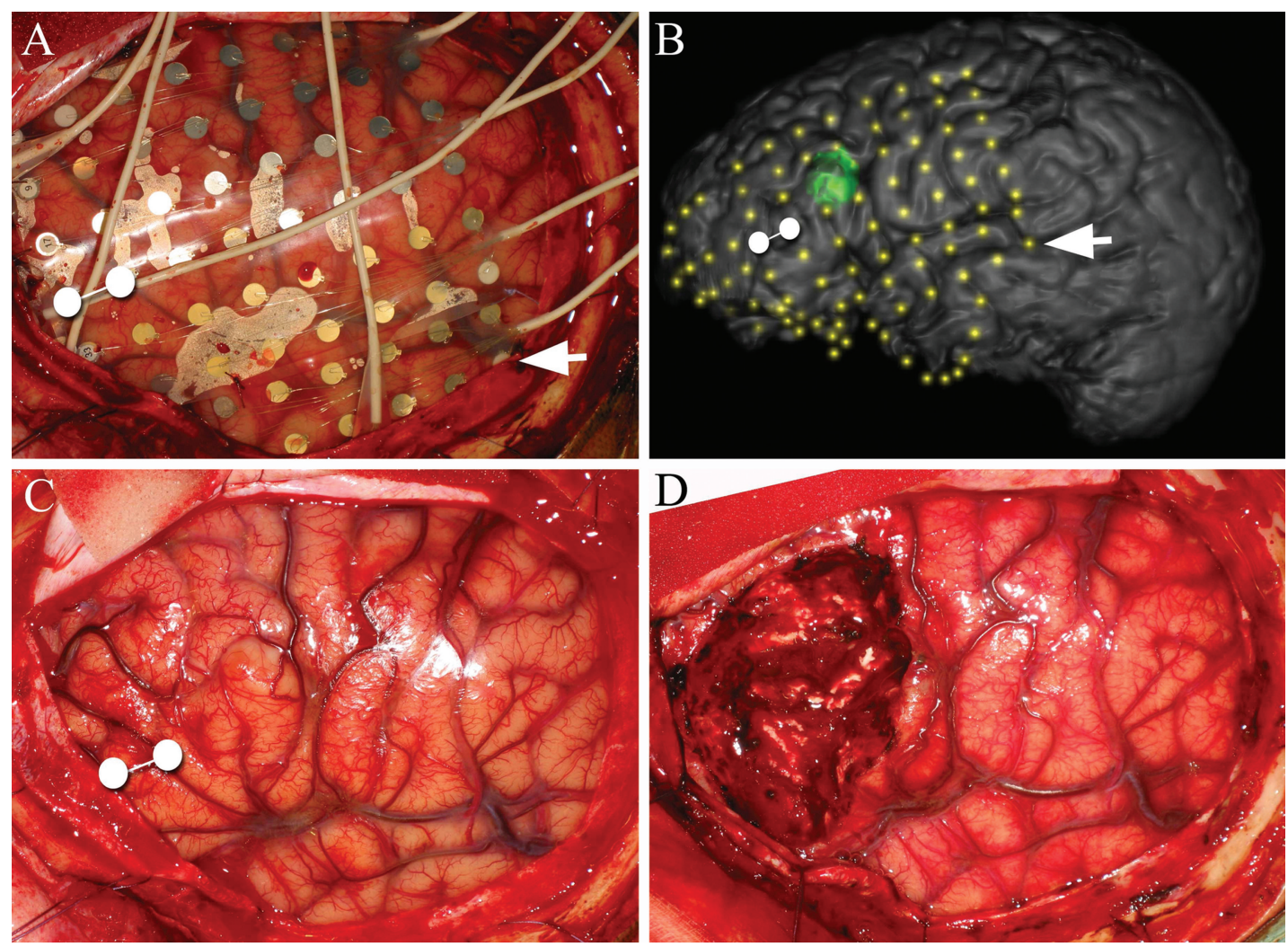

FIG. 1. A: Intraoperative photograph of left-sided frontotemporal trephination of the brain with grid electrodes in place. $\mathrm{B}$ : Volume rendering showing the cortical lesion (green area) and grid electrodes (yellow dots). The white arrows in A and $\mathrm{B}$ point to the same electrode. $\mathrm{C}$ and $\mathrm{D}$ : Intraoperative photographs before $(\mathrm{C})$ and after (D) resection. The white bar in $\mathrm{A}-\mathrm{C}$ indicates the site (electrode pair 25-26) where electrocortical stimulation disrupted working memory function.

\section{Case Report}

Summary of Case. This 8-year old right-handed girl had suffered from medically intractable partial and secondary generalized seizures since the age of 3 years. She was admitted to the Dutch Collaborative Epilepsy Surgery Program to evaluate the possibility of undergoing epilepsy surgery. Seizures were characterized by a staring spell with head and eye deviation to the right, bimanual automatisms, and postictal dysphasia. She experienced frequent seizures (5-100 per day) that interfered with school performance. Her neurological evaluation was normal. Formal neuropsychological testing of the patient was compromised by high seizure activity, which presumably influenced her scores on the Wechsler Intelligence Scale for Children-Revised (Dutch version): verbal IQ 75, performance IQ 50, and full scale IQ 60. A unilateral intracarotid artery amobarbital (or Wada) test indicated left hemisphere language involvement; MR imaging, source localization determined by magnetoencephalography and electroencephalography, and positron emission tomography indicated a left inferior frontal cortical lesion. Intracranial monitoring with grid electrodes was used for precise demarcation of functionally important areas, especially Broca's language area and the ictal onset region (Fig. 1A-C).

The invasive recording indicated a seizure onset location anterior to the left frontal lesion. Consequently, lesionectomy was performed, along with a corticectomy in the dorsolateral prefrontal cortex. This resected area left critical language sites identified with ESM intact, but included a working memory area that was identified with fMR imaging and ESM (Figs. 1 and 2). Two years after the operation she remains seizure free.

Functional MR Imaging. The patient participated in a research protocol for motor, language, and working memory function, for which the patient's parents gave written informed consent. For working memory function, a modified Sternberg Item-Recognition task was used, described in detail elsewhere. ${ }^{10,16,22}$ In brief, the patient was visually instructed to memorize a target set of five consonants. She was then presented with a series of single consonants and was instructed to press a button when she recognized a consonant belonging to the memorized target set. Conditions differed only with regard to the stimuli: a condition with practiced stimuli and a condition with novel stimuli (a higher working memory load). For the blood oxygen level-dependent (BOLD)-sensitive image acquisition, the three-dimensional navigated PRESTO pulse sequence (principles of echo shifting with a train of observations) was used as previously reported. ${ }^{18,20}$ Functional MR images were obtained with the following parameter settings: TE $35.6 \mathrm{msec}$, TR $24.6 \mathrm{msec}$, flip angle $10.5^{\circ}$, field of view $256 \times 120 \times$ $208 \mathrm{~mm}$, voxel size $4.0 \mathrm{~mm}$ isotropic, and total scan time 17.9 minutes (384 dynamic scans).

Electrocortical Stimulation Mapping. A total of 122 subdural grid electrodes (Ad-Tech) with an interelectrode spac- 

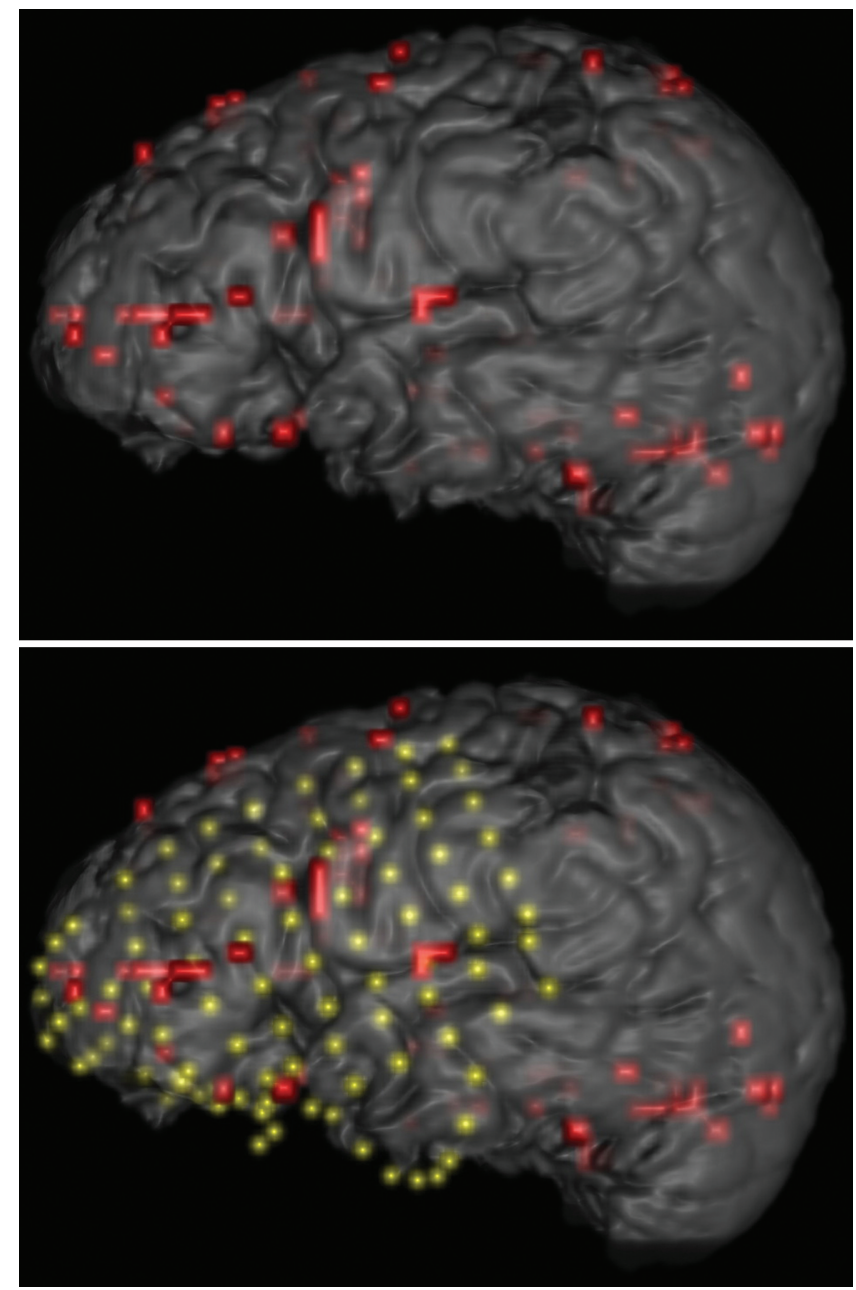

FIG. 2. Upper: Volume rendering of the brain showing fMR imaging activity $(\mathrm{T}>3.5$, red voxels) for the working memory task (practiced stimuli versus control condition). Lower: Volume rendering of the brain showing fMR imaging activity in relation to the subdural electrodes (see also Fig. 1).

ing of $1 \mathrm{~cm}$ were implanted for chronic corticographical monitoring (Fig. 1A and B). The ESM procedure was conducted using a Grass stimulator (Astro-Med) with 5-second trains of $50 \mathrm{~Hz}$ in $0.2-\mathrm{msec}$ biphasic square pulses at 5 to 8 $\mathrm{mA}$ between adjacent electrode pairs. No afterdischarges were produced. Stimulation was applied between both horizontal and vertical pairs, and also diagonally. ${ }^{11}$ For clinical purposes, motor and language areas were mapped according to previously described protocols. ${ }^{20}$ We performed a working memory task consisting of a total of 25 sequences of three letters, 15 of which coincided with electrocortical stimulation on sites of the dorsolateral prefrontal cortex. These sites were chosen on the basis of fMR imaging activity (Figs. 1 and 2). Working memory was assessed by requiring the patient to return the three letters (verbally) in reverse. An equivalent serial order task was used as a control task (comparison of digit span backward and digit span forward). We thus verbally presented sequences of letters that the patient had to rename aloud in the same (forward) or in the reverse (backward) order.

First, the baseline error-free backward span in this girl

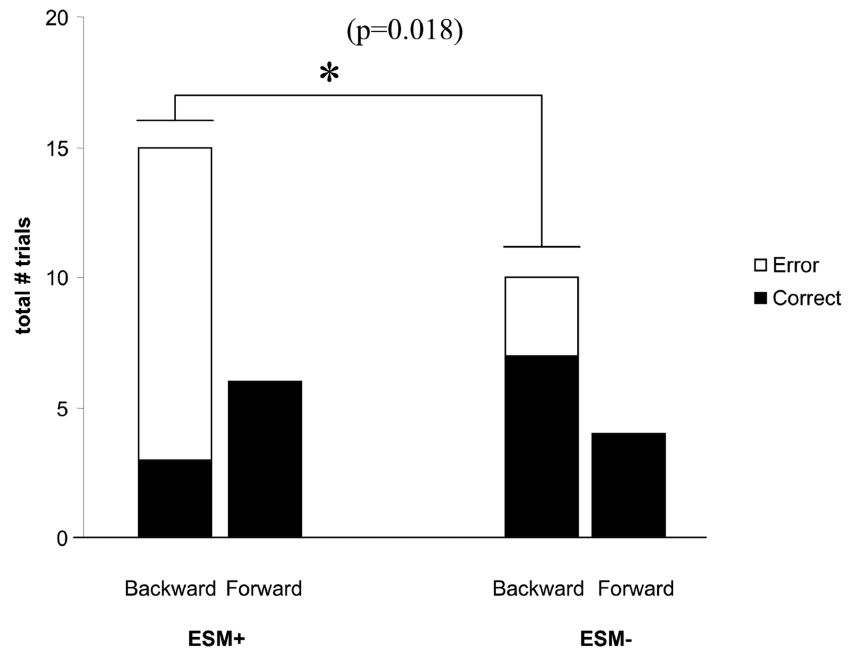

FIG. 3. Bar graph showing a significant difference in patient performance on the letterspan task with $(\mathrm{ESM}+)$ and without $(\mathrm{ESM}-)$ cortical stimulation. Electrocortical stimulation at electrode pair 2526 (indicated by the white bar in Fig. 1A-C) resulted in a significantly higher error rate during letter naming in reverse $(p=0.018$, one-sided Fisher test). Backward = naming three letters in reverse order; Forward $=$ repeating three letters in the correct order.

was established. Next, sets of three letters were presented in a series of 5 sets. Electrocortical stimulation mapping was applied during presentation of a letter sequence in three out of five sets in a blinded fashion to both the presenter and the patient. ${ }^{17}$ The difference between the number of errors in the stimulated $(\mathrm{ESM}+)$ and unstimulated $(\mathrm{ESM}-)$ condition was analyzed using the Fisher exact test.

Healthy Controls. For comparison, the reverse letter span task was performed in four age-matched healthy controls (mean age 8.7 years) who were retested after 1 week. An interaction analysis was performed with time as the withinsubject factor and group (patient versus controls) as the between-subjects factor.

Stimulation and Memory Results. An exact match was found between the fMR imaging-determined focus of working memory in the left dorsolateral prefrontal cortex and the site where stimulation significantly $(p=0.018$, one-sided Fisher exact test) interfered with performance of the backward letterspan test (Figs. 1 and 2). Stimulation at this site did not interfere with letterspan forward, indicating that stimulation did not affect task comprehension or registration of the presented letters (Fig. 3). The ESM at other electrodes, for example on sites where language disruption was found, did not affect performance of either the backward or forward task. Stimulation at the working memory site did not interfere with picture naming, reading, or spontaneous speech. The patient did not experience any distracting pain or paresthesia from stimulation. There were no afterdischarges.

This patient has remained seizure free for 2 years. Postoperative neuropsychological assessment clearly shows an increase in general performance (6-month postoperative testing, verbal IQ 94, performance IQ 79, and full scale IQ 85; 1-year postoperative testing, verbal IQ 89, performance IQ 96, and full scale IQ 91), which was also confirmed by parental observation of the patient's overall daily life and behavior, as well as her teacher's observation of school per- 
formance. Yet they noticed that she was particularly below the level of her other cognitive skills when she had to calculate with more than two numbers (requiring multiple steps to hold previous steps in working memory). Consistent with this observation, her working memory test results on the Wechsler Intelligence Scale for Children-Revised (arithmetic and digit span) continue to be below average. Her school performance improved greatly in all areas (notably language), except for arithmetic specifically. Her math performance improved slightly with remedial teaching, but remains a year behind that of her peers and remains poor even within that level.

On three different occasions after the operation, the patient was tested with the reverse letterspan task (which was also used before the operation) after 1 week, 3 weeks, and 1 year. The results of this task showed a significant deterioration, as performance dropped from $67 \%$ correct answers (before the operation) to $37 \%$ ( 1 week after the operation) (Fig. 4) (Pearson $\chi^{2}=5.45$, degrees of freedom $=1, p=0.027$, two-sided test). Compared with healthy controls in this task, her preoperative performance was normal, but decreased below the normal range postoperatively. A test-retest analysis showed that there was a significant interaction over time $(F[1,3]=$ 19.7, $p=0.021$ ), indicating improvement of performance in healthy controls after repeated testing, whereas the patient's performance significantly worsened (Fig. 5).

\section{Discussion}

This case presents evidence that ESM, the current gold standard for localizing sensorimotor and language areas, can selectively identify cortical areas involved in working memory. The relationship of ESM to fMR imaging findings was noted previously, ${ }^{17}$ but the postoperative deficit in this case strongly indicates the clinical relevance of delineating working memory areas.

The ESM procedure is performed to map critical sensorimotor and language cortex areas. To prevent postoperative aphasia, a language site detected by ESM should generally be spared during resection. Some investigators advocate

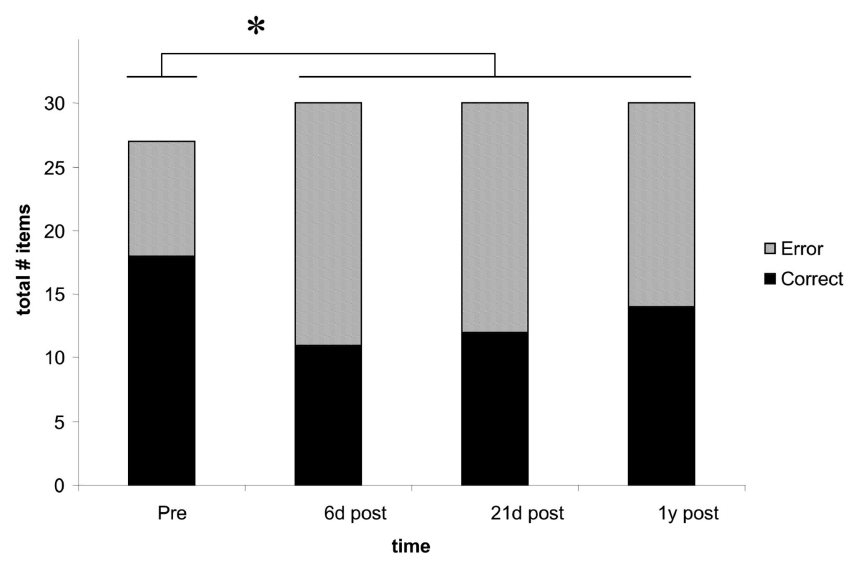

FIG. 4. Bar graph showing significant deterioration on the working memory task after resection of a left frontal lesion in the patient (Pearson $\chi^{2}$ test $=5.45$, degrees of freedom $=1, p=0.027$, twosided test). Performance on the reversed three-letterspan task is shown before the operation (pre), and on three occasions postoperatively ( 6 days, 21 days, and 1 year).

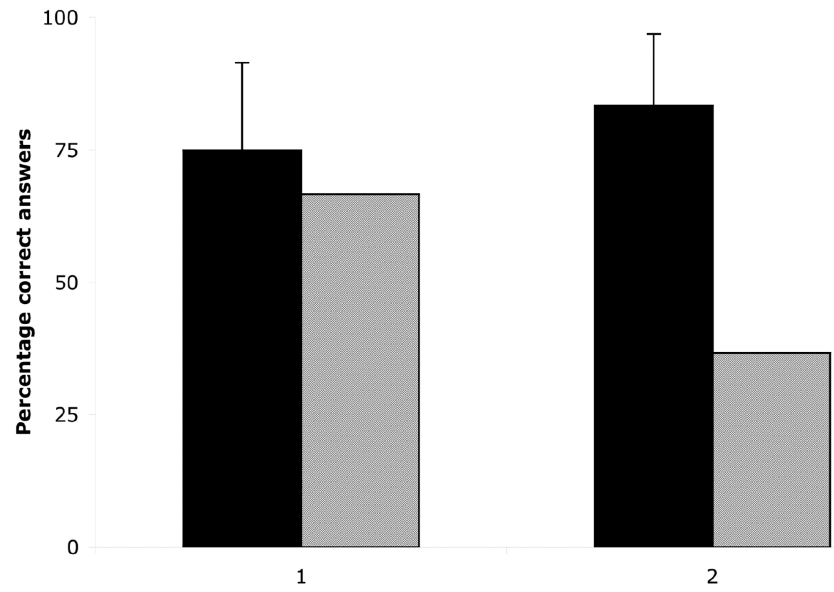

FIG. 5. Bar graph showing preoperative (1) performance of the patient (gray bar) on the reverse three-letterspan task within the range of performance of a group of four age-matched controls (black). The 1-week postoperative (2) performance significantly decreased compared with the increase in the control group $(\mathrm{F}[1,3]=19.7, \mathrm{p}=$ 0.021).

a margin of at least $1 \mathrm{~cm}$ between ESM-detected language sites and the border of the resection area. ${ }^{9}$ On the other hand, there is evidence that removal of an ESM-detected language area does not always induce postoperative aphasia, a finding that calls into question the clinical relevance of ESM. ${ }^{21}$

For obvious ethical reasons, no one but Seeck and colleagues ${ }^{21}$ has described deliberate resection of ESM-detected language areas, and to our knowledge no data on other domains of higher cognition are available. In our case the epileptogenic region overlapped the identified working memory site, thus posing an ethical problem. Considering that the epileptogenic region was outside the classical critical motor and language cortex and given the experimental nature of our working memory protocol, the clinical decision was made to include the presumed working memory area in the resection. This decision resulted in a seizure-free outcome and a general improvement in the patient's daily function and quality of life, but also in a postoperative impairment of working memory, implying that the ESM site is critical for working memory performance. Because Broca's area did not overlap the working memory site and postoperative language testing showed improvement in language tasks, such as repeating long sentences, our specific test may not cover all aspects of working memory. Indeed, working memory needed for sentences may be dissociated from working memory as measured by tests comparable to ours ${ }^{3,4}$ and may not be dependent on the dorsolateral prefrontal cortex site identified in this study.

Through the follow-up years, there was some improvement in the patient's performance of the letterspan backward test, and her general cognitive performance has improved considerably. Plasticity of the brain has been demonstrated after hemispherectomy ${ }^{5}$ and after tumor surgery ${ }^{6}$ in which the initial decline of function is followed by recovery. Postoperative recovery of function has been associated with more activation in the healthy contralateral analogue of the resected brain area. ${ }^{12}$ It is understood that working memory function relies on a network of multiple cortical areas,,$^{10}$ which may be crucial to rehabilitation of the impaired function. 


\section{Working memory deficits after resection predicted by fMRI and ESM}

This study does not indicate conclusively whether the dorsolateral prefrontal cortex is critical for working memory, but the selective decrease in math performance and the disproportionately slow recovery in this patient strongly suggest that this is true.

Further research in more patients should reveal whether the standard clinical workup for identifying critical areas (language and sensorimotor function) should be extended with tests of other cognitive functions such as working memory. These data compel us to consider clinical and ethical dilemmas, which also arose in this case. Developing new methods and tools to identify other (cognitive) functions than language and motor skill is essential to improving a patient's postoperative quality of life. We continue to work to assess the specific cognitive functions that are subserved by the dorsolateral prefrontal cortex in other patients, using grid electrodes positioned over this region.

\section{References}

1. Baddeley AD: Working Memory. Oxford: Oxford University Press, 1986

2. Blanke O, Ortigue S, Landis T, Seeck M: Stimulating illusory own-body perceptions. Nature 419:269-270, 2002

3. Caplan D, Waters G: Short-term memory and language comprehension: a critical review of the neuropsychological literature, in Vallar G, Shallice T (eds): Neuropsychological Impairments of Short-Term Memory. Cambridge: Cambridge University Press, 1990, pp 337-389

4. Caplan D, Waters GS: Verbal working memory and sentence comprehension. Behav Brain Sci 22:77-126, 1999

5. de Bode S, Curtiss S: Language after hemispherectomy. Brain Cogn 43:135-138, 2000

6. Duffau H: Lessons from brain mapping in surgery for low-grade glioma: insights into associations between tumor and brain plasticity. Lancet Neurol 4:476-486, 2005

7. Goldman-Rakic PS: The prefrontal landscape: implications of functional architecture for understanding human mentation and the central executive. Philos Trans R Soc Lond B Biol Sci 351:1445-1453, 1996

8. Goldman-Rakic PS: Regional and cellular fractionation of working memory. Proc Natl Acad Sci U S A 93:13473-13480, 1996

9. Haglund MM, Berger MS, Shamseldin M, Lettich E, Ojemann GA: Cortical localization of temporal lobe language sites in patients with gliomas. Neurosurgery 34:567-576, 1994

10. Jansma JM, Ramsey NF, Slagter HA, Kahn RS: Functional anatomical correlates of controlled and automatic processing. J Cogn Neurosci 13:730-743, 2001

11. Kho KH, Leijten FS, Rutten GJ, Vermeulen J, Van Rijen P, Ramsey NF: Discrepant findings for Wada test and functional magnetic resonance imaging with regard to language function: use of electrocortical stimulation mapping to confirm results. Case report. J Neurosurg 102:169-173, 2005

12. Krainik A, Lehericy S, Duffau H, Capelle L, Chainay H, Cornu P, et al: Postoperative speech disorder after medial frontal surgery: role of the supplementary motor area. Neurology 60:587-594, 2003

13. Leijten FS, Ramsey NF, Van Rijen PC: Function localisation in neurosurgery: new tools, new practices? Clin Neurol Neurosurg 107:17-19, 2004

14. Ojemann GA: The neurobiology of language and verbal memory: observations from awake neurosurgery. Int J Psychophysiol 48:141-146, 2003

15. Penfield W: Some mechanisms of consciousness discovered during electrical stimulation of the brain. Proc Natl Acad Sci U S A 44:51-66, 1958

16. Ramsey NF, Jansma JM, Jager G, Van Raalten T, Kahn RS: Neurophysiological factors in human information processing capacity. Brain 127:517-525, 2004

17. Ramsey NF, Kho KH, Leijten FS, van Rijen P, van der Schaaf A: Cross-validation of localized working memory function between fMRI, cortical electrostimulation and subdural EEG mapping. Neuroimage 22: E837-e838, 2004

18. Ramsey NF, van den Brink JS, van Muiswinkel AM, Folkers PJ, Moonen CT, Jansma JM, et al: Phase navigator correction in 3D fMRI improves detection of brain activation: quantitative assessment with a graded motor activation procedure. Neuroimage 8:240-248, 1998

19. Roux FE, Boetto S, Sacko O, Chollet F, Tremoulet M: Writing, calculating, and finger recognition in the region of the angular gyrus: a cortical stimulation study of Gerstmann syndrome. J Neurosurg 99:716-727, 2003

20. Rutten GJ, Ramsey NF, van Rijen PC, Noordmans HJ, van Veelen $\mathrm{CW}$ : Development of a functional magnetic resonance imaging protocol for intraoperative localization of critical temporoparietal language areas. Ann Neurol 51:350-360, 2002

21. Seeck M, Pegna AJ, Ortigue S, Spinelli L, Dessibourg CA, Delavelle J, et al: Speech arrest with stimulation may not reliably predict language deficit after epilepsy surgery. Neurology 66: 592-594, 2006

22. Sternberg S: High-speed scanning in human memory. Science 153:652-654, 1966

Manuscript submitted June 7, 2006.

Accepted November 30, 2006.

This work was supported by the Dutch Epilepsy Foundation, Grant No. 01-04 (K.H.K.) and the Netherlands Organisation for Scientific Research (NWO), VIDI Grant No. 917.36.401 (N.F.R.).

Address reprint requests to: Kuan H. Kho, M.D., Department of Neurosurgery, University Medical Center Utrecht, P.O. Box 85500, 3508 GA Utrecht, The Netherlands. email: k.h.kho@umcutrecht.nl. 